Orthopäde $2011 \cdot 40: 377$

DOI 10.1007/s00132-010-1718-x

Online publiziert: 8. April 2011

(c) Springer-Verlag 2011

\author{
N. Wülker \\ Orthopädische Klinik, Universitätsklinikum Tübingen
}

\title{
Fußchirurgie - Probleme und Komplikationen vermeiden!
}

Sehr geehrte Leserinnen und Leser,

in der Fußchirurgie sind Probleme und Komplikationen insgesamt nicht häufiger als anderswo in der Orthopädie und Unfallchirurgie. Eine Komplikationsrate von einigen Prozent lässt sich auch bei noch so guter Operationstechnik nicht vermeiden. Allerdings beobachtet man eine manchmal recht unterschiedliche Komplikationsquote innerhalb der Fußchirurgie, wenn man sich dort intensiv umsieht.

Immer ist es dennoch möglich, durch Details der Operationstechnik die Rate von Problemen und Komplikationen noch weiter günstig zu beeinflussen. Der Fuß ist empfindlicher als die meisten anderen Gelenke oder Organe. Dies liegt an der dünnen Weichteildeckung, da der Fuß im Wesentlichen nur aus Haut, Knochen und Sehnen besteht. Außerdem ist der Fuß weiter als jeder andere Körperteil von Herz und Gehirn entfernt. Erkrankungen der Gefäße und Nerven treten daher am Fuß früher in Erscheinung als anderswo. Man erkennt sie nur, wenn man gezielt danach fragt und untersucht. Probleme und Komplikationen werden also bereits vor der Operation vermieden, indem man die Indikation korrekt stellt bzw. insbesondere eben auch manchmal nicht stellt.

\section{Die Operationstechnik ent- scheidet am Fuß mehr als an manchen anderen Körper- regionen über das Ergebnis}

Auch die Operationstechnik innerhalb der Fußchirurgie unterscheidet sich er- heblich, je nachdem welchen Fußchirurgen man besucht. Und es führen ja auch viele Wege nach Rom. Immer ist es besser, wenn ein Chirurg einen Eingriff so gut macht, wie er ihn gelernt hat, mit Sicherheit besser, als wenn eine andere Technik gemacht aber nicht beherrscht wird. Einige Prinzipien in der Fußchirurgie erfordern dennoch besondere Beachtung. Hier geht es insbesondere um eine Gewebe schonende Operationstechnik, die hilft, Wundheilungsstörungen zu vermeiden. Manchmal fällt es schwer, sich von lieben Gewohnheiten, z. B. dem Elektrokauter, zu verabschieden.

Und auch nach der Operation werden Probleme und Komplikationen durch eine sachgerechte Technik vermieden. Hier geht es besonders um die Verbandstechnik, die Kontrolle der Durchblutung und eine möglichst funktionelle Nachbehandlung, die einer Gewebeatrophie vorbeugt. Erst wenn es auch hier optimal läuft, rundet sich das Bild einer optimalen Fußchirurgie ab.

Manche Eingriffe am Fuß entsprechen technisch betrachtet vergleichbaren Operationen an anderen Körperregionen, z. B. Endoprothesen am Großzehengrund- und am oberen Sprunggelenk. Andere Eingriffe folgen speziellen Prinzipien, wie es sie so nur am Fuß gibt, beispielsweise die Triplearthrodese oder Resektionsarthroplastiken am Vorfuß. Diese speziellen Techniken müssen erlernt werden, da man die dort gestellten Anforderungen nicht mit einem Basiswissen der Orthopädie und Unfallchirurgie allein erfüllen kann.
Brauchen wir also einen spezialisierten Fußchirurgen? Ich meine nein. Nur wer in der Orthopädie allgemein mit Gewebe, Knochen und Gelenken gelernt hat umzugehen, kann dieses Wissen produktiv am Fuß umsetzen. Im internationalen Vergleich haben wir im deutschsprachigen Raum sehr gut und vor allem sehr breit ausgebildete Chirurgen in der Orthopädie und Unfallchirurgie. Wenn diese Orthopäden und Unfallchirurgen im Rahmen der Weiterbildung oder der Fortbildung gelernt haben, mit den speziellen Herausforderungen der Fußchirurgie umzugehen, ist dies die optimale Voraussetzung für eine hoch qualifizierte Versorgung der Patienten.

Ihr

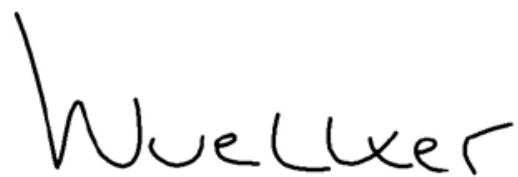

N. Wülker

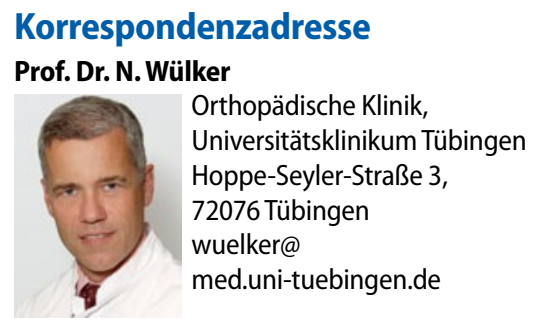

Interessenkonflikt. Der korrespondierende Autor gibt an, dass kein Interessenkonflikt besteht. 